\title{
Effects of pre-drying on quality of fried breaded black pomfret (parastromateus niger)
}

fillet.

\begin{abstract}
The objective of this study was to investigate the effects of pre-drying process on quality of fried breaded fish fillets. For this study, breaded black pomfret (Parastromateus niger) fillets were pre-dried in conventional oven at $180^{\circ} \mathrm{C}$ for $0,30,60,90$ and $120 \mathrm{sec}$. The pre-dried fillets were pre-fried in sunflower oil and stored at $-20^{\circ} \mathrm{C}$ for 1 week. They were then finally cooked in the combination oven. Fat, moisture, texture and color of the cooked fillets were evaluated. Results indicated that moisture loss and the fat uptake of cooked fillets decreased with increasing pre-drying time. Instrumental texture analysis showed that hardness of the pre-dried samples increased as compared to the control. Results from color evaluation showed that the $b^{*}$ (yellowness) values of the samples increased, while $\mathrm{L}^{*}$ (whiteness) and $\mathrm{a}^{*}$ (redness) values did not change significantly $(\mathrm{p}<0.05)$. The best quality product was prepared when 90 sec pre-drying time was applied.
\end{abstract}

Keyword: Pre-drying; Sunflower oil; Oven cooking; Quality characteristics. 